Trauma Berufskrankh 2004 · 6 [Suppl 4] : S408-S416 DOI 10.1007/s10039-003-0805-1

Online publiziert: 23. Dezember 2003

(c) Springer-Verlag 2003

M. Beck $\cdot$ T. MittImeier

Abteilung für Unfall- und Wiederherstellungschirurgie der Klinik und Poliklinik für Chirurgie,

Universität Rostock, Rostock

\title{
Spezielle Probleme und Spätfolgen nach OSG-Fraktur
}

tibiotarsale oder tibiometatarsale Transfixation, ggf. dreidimensional unter Einbeziehung des Fersenbeins, erfolgen. Gleiches Vorgehen empfiehlt sich bei Frakturen mit bereits bestehenden manifesten Weichteilproblemen in Form von stark geschwollenen Weichteilen, Spannungsblasen oder Kontusionszonen. Dieses Konzept erleichtert die weitere Weichteilbeurteilung und -pflege. Das verletzte Sprunggelenk ist frei zugängig und es können effektiver abschwellende Maßnahmen (Kühlung, AV-Pumpe) durchgeführt werden. Die korrekte Achseneinstellung verhindert Drucknekrosen der Haut, führt weiterhin zu einer forcierten posttraumatischen Erholung des Weichteilmantels und verhindert sekundäre Weichteilschäden durch Gipsdruck von außen. Die befürchtete erhöhte Infektgefahr durch die eingebrachten Fixateur-Pins konnten wir bei diesem Vorgehen im eigenen Krankengut nicht beobachten.

\section{Kompartmentsyndrom}

Das Kompartmentsyndrom nach OSGFraktur ist ein sehr seltenes Ereignis. In der Literatur finden sich nur vereinzelte Fallberichte [7]. Besteht jedoch der klinische Verdacht, muss eine umgehende chirurgische Intervention erfolgen. Neben der kompletten Eröffnung der 4 Unterschenkelkompartimente müssen die Retinakula der 3 Sehnengruppen (Extensoren-, Flexoren- und Peroneussehnen) ge- spalten werden und die Spaltung bei entsprechender Klinik auf die Kompartmenträume des Fußes ausgeweitet werden. Eine operative Stabilisierung durch internes oder externes Verfahren sollte in jedem Fall in gleicher Sitzung erfolgen.

\section{Fehlerhafte Osteosynthesen, operativ fixierte Fehlstellungen}

Fehlerhafte Osteosynthesen durch ungeeignete Implantate sollten grundsätzlich vermeidbar sein. Deutsche und internationale Gesellschaften haben klare und verbindliche Behandlungsrichtlinien zur Behandlung der OSG-Fraktur erarbeitet $[16,19]$. Es ist der Verdienst der Arbeitsgemeinschaft für Osteosynthesefragen, die Standardimplantate mitentwickelt und etabliert zu haben [12]. Unbegründete Abweichungen führen immer wieder zu Fehlschlägen und schlechten Ergebnissen durch Implantatversagen. Die in - Abb. 1a, b gezeigte Versorgung einer Weber-C-Fraktur mit Trümmerzone und Syndesmosenverletzung mittels „Zuggurtungsosteosynthese und Hemicerclagen" ist ein völlig ungeeignetes Verfahren. Bereits die unmittelbare postoperative Röntgenkontrolle zeigt die nicht akzeptable Frakturstellung bei unversorgter Syndesmosenverletzung.

Fixierte Fehlstellungen der Fibula können in Form von Verkürzungen, Rotationsfehlstellungen und Inkongruenz in der Incisura fibularis tibiae vorliegen. Ver- 

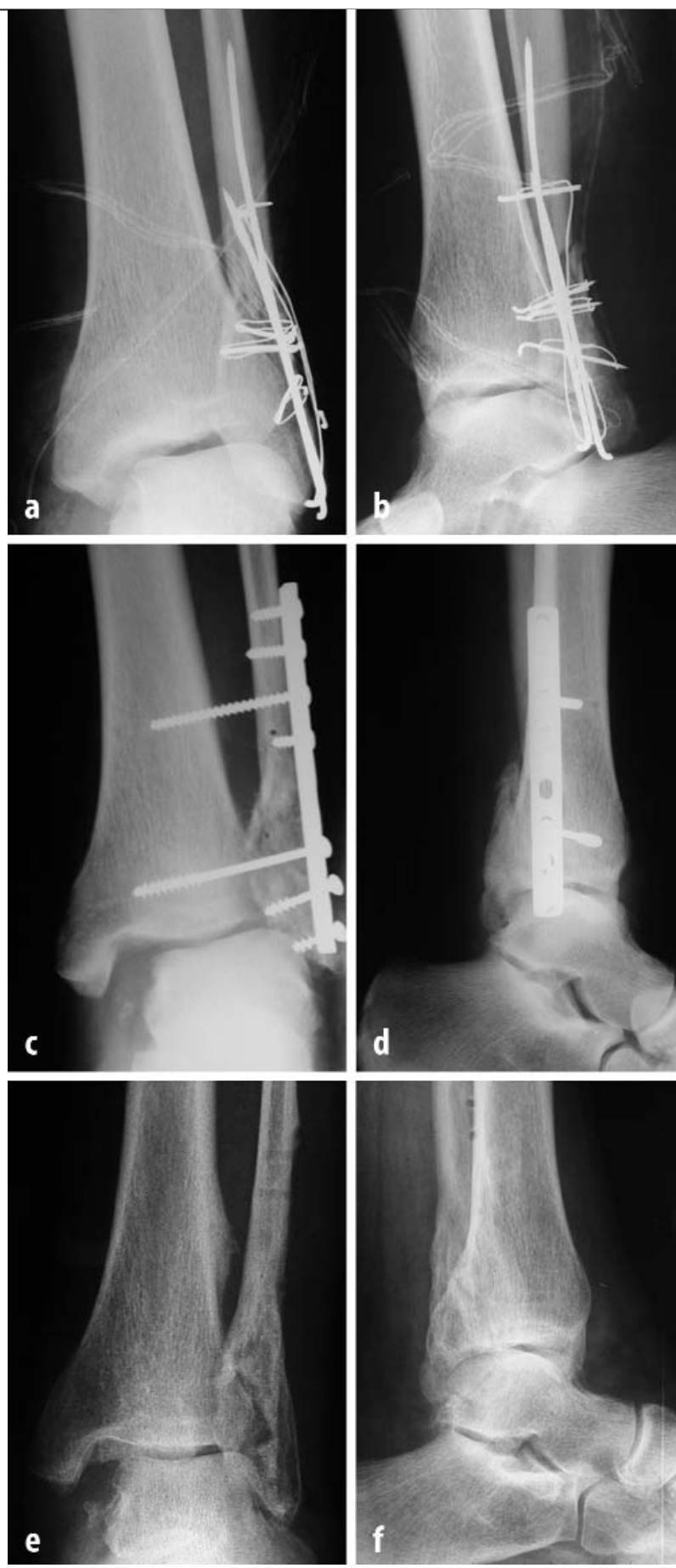

Abb. $1<a$, b Insuffiziente Primärosteosynthese einer Außenknöchelfraktur durch Zuggurtung und Hemicerclagen, nicht versorgte Syndesmosenverletzung. Kompression der Trümmerzone statt Überbrückung. $c$, $d$ Fehlerhafte Revisionsosteosynthese mit Platte und Stellschrauben, deutliche Fibulaverkürzung, medial verbreiterter Gelenkspalt, Talussubluxation bei fehlfixierter Fibula.e, f Schwere posttraumatische Arthrose 3 Jahre nach Unfall

kürzungen und Rotationsfehlstellungen sind hauptsächlich ein Problem bei Trümmerfrakturen der Fibula, die eine anatomische Rekonstruktion nicht mehr zulassen. Fehlstellungen der Fibula in der Inzisur finden sich bei nicht diagnostizierter Mitverletzung des Syndesmosenkomplexes und inkorrekt reponierter und durch Stellschraube fehlfixierter Fibula. Die postoperativen Röntgenbilder (- Abb. 1c, d) - es handelt sich um die „Korrekturosteosynthese“ der in den
Trauma Berufskrankh 2004 6 [Suppl 4] :

S408-S416

DOI 10.1007/s10039-003-0805-1

(C) Springer-Verlag 2003

\section{Beck $\cdot$ T. Mittlmeier}

\section{Spezielle Probleme und Spätfolgen nach OSG-Fraktur}

\section{Zusammenfassung}

Ziel bei der Behandlung der Frakturen des oberen Sprunggelenkes ist die Wiederlangung einer optimalen Gelenkfunktion. Prognostische Faktoren sind das Ausmaß der Verletzung, begleitende Knorpelschäden der gelenkbildenden Flächen von Fibula, Tibia und des Talus sowie Alter und Geschlecht der Patienten. Die Prognose wird aber auch entscheidend von der korrekten chirurgischen Versorgung der Frakturen bestimmt. Komplikationen wie Instabilität, Kompartmentsyndrom, Infektion, Pseudarthrosen, Arthrose und fixierte Fehlstellungen sowie Maßnahmen zu ihrer Vermeidung und Behandlung werden dargestellt.

\section{Schlüsselwörter}

Oberes Sprunggelenk · Prognose · Komplikation OSG-Fraktur

\section{Specific problems and long-term consequences after ankle fractures}

\section{Abstract}

The therapeutic goal for ankle fractures is to regain optimum function of the ankle. Prognostic factors include the degree of injury, additional lesions of the cartilage forming the joint surface of the fibula, tibia, and talus, and also the age and sex of the patient. The correct surgical treatment of the fractures, however, is crucial for the prognosis. Complications such as instability, compartment syndrome, infection, pseudarthrosis, arthrosis, and fixed malpositions are described as well as means of avoiding and treating them.

\section{Keywords}

Ankle · Prognosis · Complication · Ankle fracture 

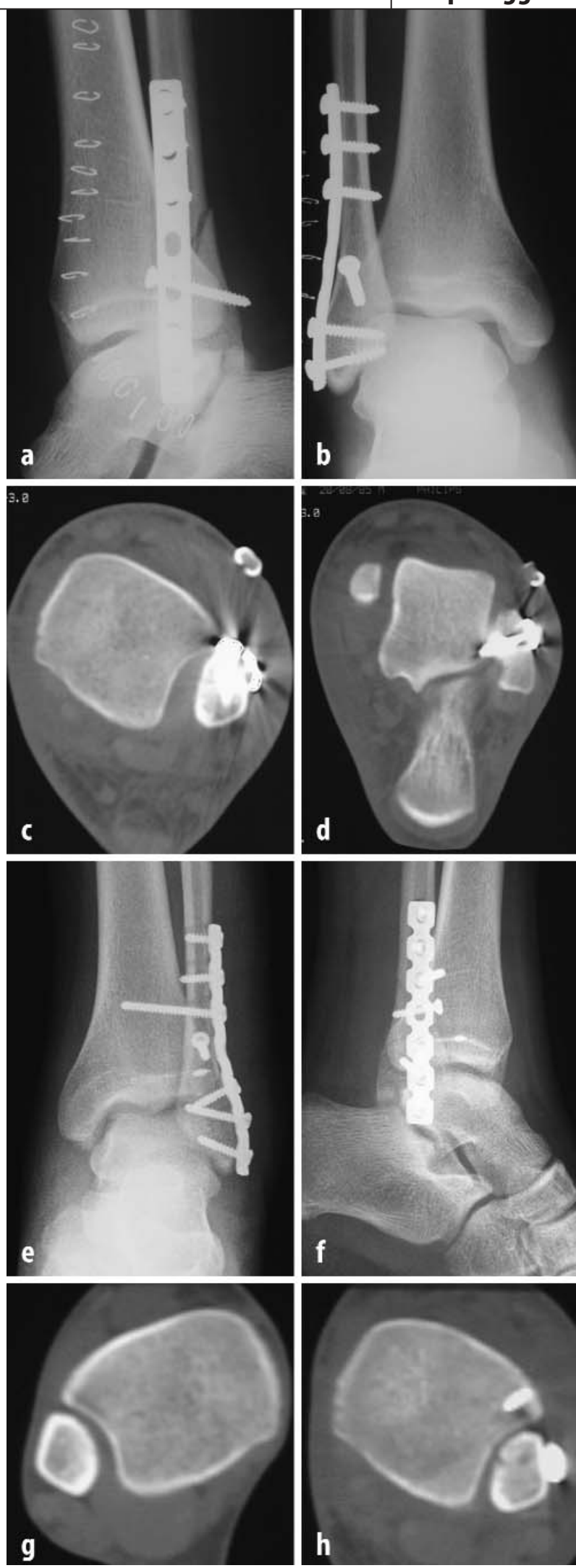

Abb. $2<a$, b Fehlerhafte Osteosynthese mit deutlich erkennbarer Stufenbildung und Fibulaverkürzung. Medial erweiterter Gelenkspalt als Hinweis auf eine unversorgte Syndesmosenverletzung. c, d Die CT vor Revision zeigt die Fehlstellung der Fibula in der Inzisur und eine überlange Schraube, welche die laterale Taluswand tangiert. e, f Revisionsosteosynthese mit Rekonstruktionsplatte, Stellschraubenimplantation und Periostlappenplastik des LTFA (Knochenankerfixation tibial). $g$, $h$ Die postoperative Kontroll-CT zeigt die nun korrekt eingestellte Fibula im Vergleich zur gesunden Gegenseite
Grundsätzlich muss bei jeder operativ versorgten Außenknöchelfraktur die Integrität des Syndesmosenkomplexes intraoperativ getestet werden. Die Inspektion des vorderen Syndesmosenbandes, die klinische Stabilitätsprüfung in ventrodorsaler Richtung, die seitengleiche Weite des medialen und lateralen $\mathrm{Ge}$ lenkspalts, der aktive Hakenzugtest der Fibula sowie die dynamische PronationsAußenrotations-Probe unter Bildwandlerkontrolle müssen standardisiert durchgeführt werden. Auch bei korrekter und vollständiger Durchführung dieser intraoperativen Tests beruht letztendlich die Einschätzung der Stabilität des Syndesmosenkomplexes auf der subjektiven Wahrnehmung und den Erfahrungswerten des Operateurs. Postoperativ ist eine Verkürzung der Fibula im konventionellen Röntgenbild anhand überlappender Stufenbildung der Fragmente, der Korrelation Außenknöchelspitze zur lateralen Talusfläche (Weber-Ball) und der „, Weber-Nase“ gut zu erkennen (• Abb. 2a, b). Die korrekte Einstellung der Fibula in der Incisura fibularis tibiae kann radiologisch jedoch nur anhand indirekter Hinweise in Sinne eines erweiterten, ,espace claire“ nach Chaput ( $>6 \mathrm{~mm}$ ) und der medialen Gabelweite abgeschätzt werden [3].

Ausreichend sicher sind fibulare Rotationsfehlstellungen und den Syndesmosenkomplex betreffende Fehlstellungen jedoch nur durch eine postoperative CT zu diagnostizieren ( $\bullet$ Abb. 2c, d). Aus diesem Grund führen wir bei Mitverletzung des Syndesmosenkomplexes und durchgeführter Stellschraubenimplantation grundsätzlich eine postoperative CT des verletzten und gesunden Sprunggelenkes durch. Hier kann die Stellung der Fibula in der Inzisur im Vergleich zur gesunden Seite kontrolliert werden und die Rotation der Fibula ermittelt werden. Um einen standardisierten CT-Untersuchungsablauf zu gewährleisten, erfolgt die Lagerung in einer von uns konstruierten röntgendurchlässigen Schiene mit flexibel einstellbaren Winkelgraden für das obere Sprunggelenk und Fixationsmöglichkeiten für Unterschenkel und Fuß (- Abb. 3).

Experimentell konnte nachgewiesen werden, dass eine Verkürzung und/oder eine Lateralisation der Fibula um $2 \mathrm{~mm}$ 
und/oder eine Außenrotation von $5^{\circ}$ bei einer simulierten Weber-C-Fraktur zu einer signifikanten Steigerung der Druckbelastung im OSG führt [18]. Somit führt die Nichtbehandlung fehlfixierter OSGFrakturen unausweichlich zur frühzeitigen posttraumatischen Arthrose. Analog zu diesen experimentellen Werten sehen wir die Indikation zur Revision bei Verkürzungen und Fehlpositionierungen der Fibula in der Inzisur von mehr als $2 \mathrm{~mm}$

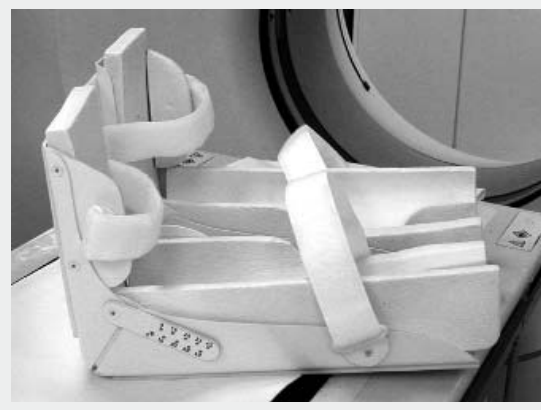

Abb. $3<$ Speziell konstruierte röntgendurchlässige Lagerungsschiene für die computertomographische Untersuchung der oberen Sprunggelenke

Abb. $4 \nabla$ a, b Trimalleoläre OSG-Fraktur, Eversions-Pronations-Typ mit Syndesmosenverletzung. Ausgeprägte Osteoporose, Gefäßverkalkungen bei pAVK. c, d Korrekt durchgeführte Osteosynthese der trimalleolären OSG-Fraktur. Wegen der ausgeprägten 0steoporose zusätzlich protektiver Fixateur externe, später Orthesenstiefel. e, f Sekundäre Dislokation durch unerlaubte Vollbelastung, zudem begleitendes sequestrierendes Infektgeschehen. $g$, h Zustand nach kompletter Implantatentfernung, Sequestrektomie, liegende Vakuumversiegelung und Früharthrodese durch llizarov-Fixateur
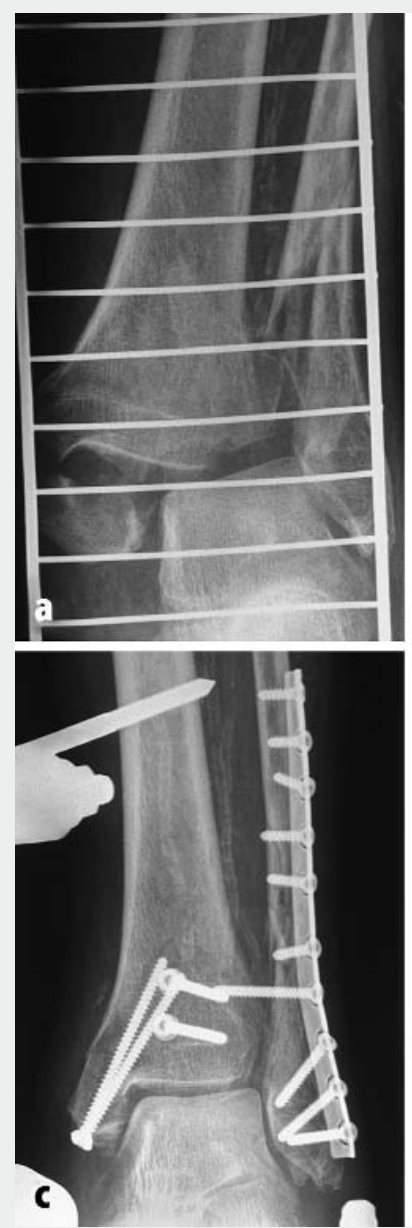
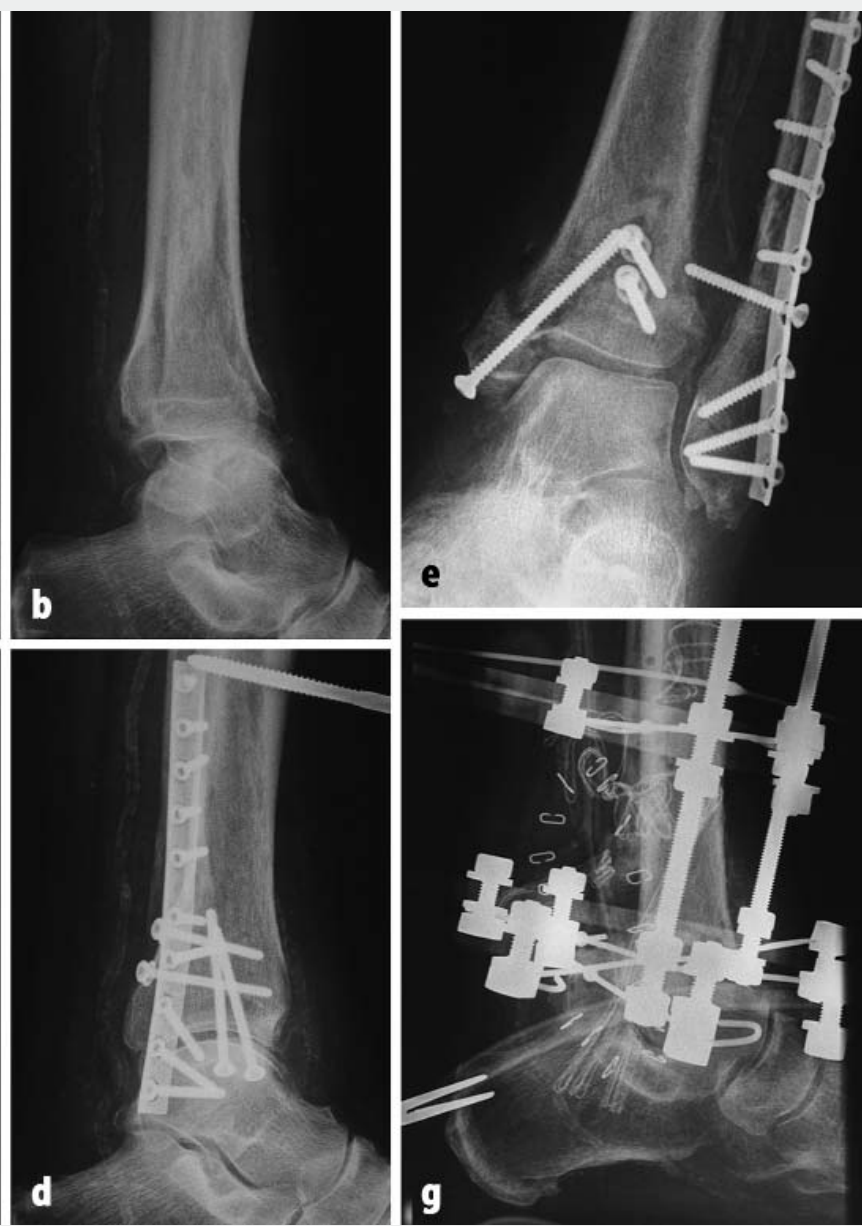

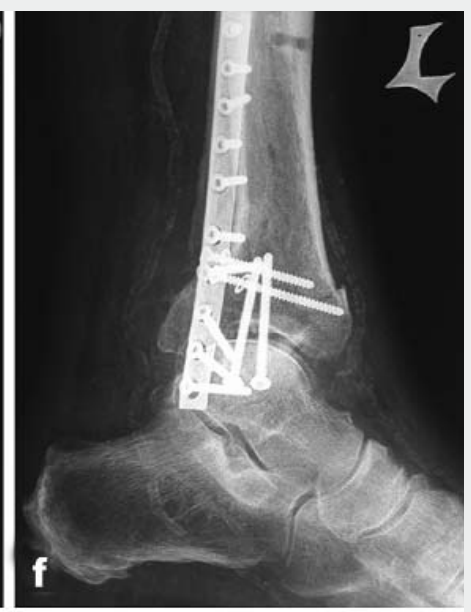

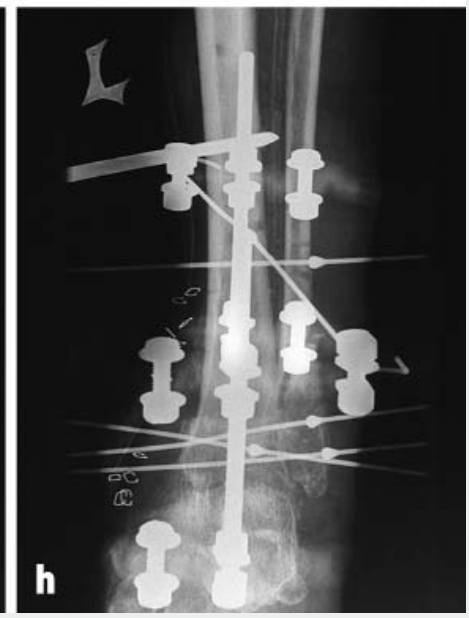



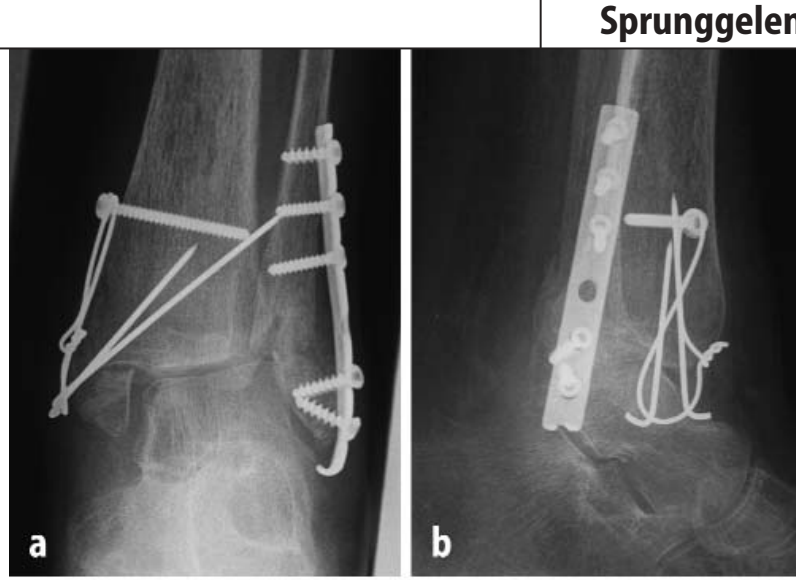
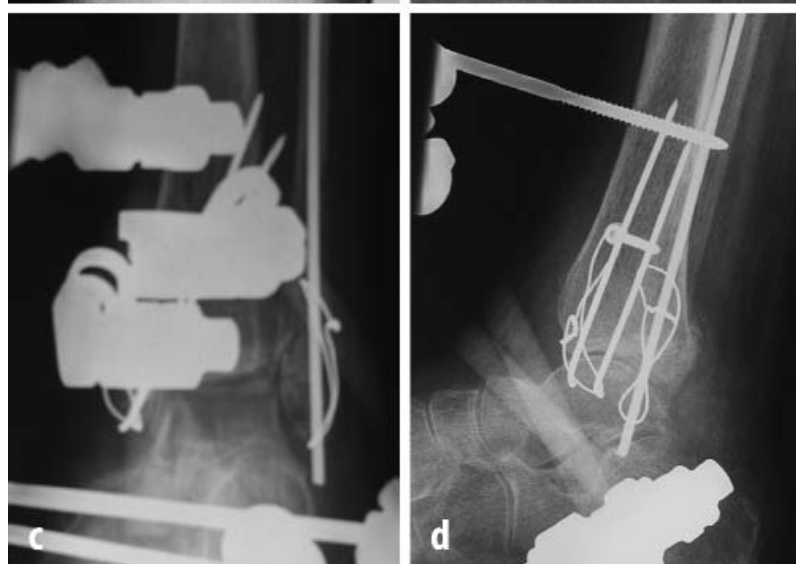

Abb. $5<$ a, b Osteosynthesematerialausbruch bei bimalleolärer OSG-Fraktur, Innenknöchelzuggurtung inkorrekt, ausgeprägte Osteoporose. c, d SalvageOsteosynthese nach kompletter Implantatentfernung durch Anlage eines dreidimensionalen Fixateur externe, intramedullären Prevotnagels und Zuggurtung der Fibula sowie Neuanlage der Innenknöchelzuggurtung

hier ist nach erfolgter Revision des Außenknöchels und Versorgung einer unteren Syndesmosenverletzung die postoperative Computertomographie in unseren $\mathrm{Au}$ gen obligat (• Abb. 2g, h).

Nicht korrekt reponierte Innenknöchelfrakturen, die eine zusätzliche Impressionszone aufweisen, bereiten gelegentlich erhebliche Schwierigkeiten der korrekten Einstellung, da die Fragmentränder keine zuverlässigen Referenzpunkte mehr bieten. Bestehen Impressionszonen am Übergang zur Pilongelenkfläche, müssen diese angehoben werden und entsprechend unterfüttert werden. Die Innenknöchelversorgung erfolgt dann durch Zugschrauben oder bei zu großem Substanzverlust durch die Voroperation durch Zuggurtung.

Für eine suffiziente Versorgung der Kantenfragmente müssen ebenfalls Kleinfragmentschrauben verwendet werden. Eine indirekte, wenn auch radiologisch kontrollierte Reposition des hinteren Volkmann-Dreiecks birgt durch Zug der ansetzenden Bänder die Gefahr der Verkippung und der Stufenbildung. Fragmente mit relevanten Gelenkflächenanteilen sollten daher offen unter Sicht anatomiege- recht reponiert werden und durch direkte posteriore oder indirekte anteriore Verschraubung versorgt werden.

\section{Sekundäre Dislokation/ Salvage-Verfahren}

Ursachen für das Versagen einer korrekt durchgeführten Osteosynthese (- Abb. 4a-d) sind v. a. eine vorzeitige übermäßige Belastung des operierten Sprunggelenkes und das Vorliegen einer ausgeprägten Osteoporose. Weitere Risikofaktoren sind eine begleitende symptomatische pAVK und der Diabetes mellitus, die zu einer verzögerten Knochenheilung beitragen können.

Protektive Maßnahmen in Form einer zusätzlichen Cast-Ruhigstellung oder eines „Orthesen-Schuhs“ können das Risiko reduzieren, aber nicht vollständig beseitigen (• Abb. 4e, f).

Der Versuch der standardisierten Reosteosynthese ist oft zum Scheitern verurteilt, da durch den Ausbruch des primären Osteosynthesematerials die ohnehin schon osteoporotische Knochenstruktur zusätzlich geschädigt ist. Weiterhin liegen bei diesen Risikopatienten oft prekäre Weichteilverhältnisse vor, sodass in diesen seltenen Fällen auf unkonventionelle Verfahren zurückgegriffen werden muss. Erste Option ist nach Entfernung des dislozierten Osteosynthesematerials die Anlage eines Fixateur externe. Über diesen kann eine korrekte Stellung des Talus zur tibialen Gelenkfläche erzielt werden sowie Länge und Rotation des Sprunggelenkes weitgehend korrekt eingestellt werden. Zusätzliche intramedulläre Implantate eignen sich gut, um eine zusätzliche Adaptation und Stellungsverbesserung von Außen- und Innenknöchel zu erreichen (• Abb. 5a-d).

Gleiches Vorgehen kann primär bei kritischen Weichteilverhältnissen notwendig werden. Die Kombination aus schlecht abheilenden großflächigen Spannungsblasen und Durchblutungsstörungen verbietet eine reguläre osteosynthetische Versorgung und macht die genannten Salvage-Verfahren zur korrekten Achsen- und Längeneinstellung notwen$\operatorname{dig}(\bullet$ Abb. 6a-c).

\section{Infektion}

Die Infektrate liegt bei operativ behandelten OSG-Frakturen zwischen 1 und $2 \%$ [11]. Bildet sich ein manifester Frühinfekt aus, so muss eine konsequente Therapie nach den allgemein gültigen Richtlinien der Osteitisbehandlung erfolgen. Chirurgisches Débridement, ausgiebige Spülung, intraoperative Abstrichentnahme, Antibiotikaschaum oder -ketteneinlage, längere Drainage des Wundbereichs und, wenn nötig, Etappenrevisionen sowie die Gabe keimgerechter Antibiotika sind die Grundlagen der Therapie. Es ist gerechtfertigt, gerade bei noch instabilen Verhältnissen, die Implantate zu belassen, wenn keine Hinweise auf Sequestrierung vorliegen. Bei kritischen Weichteilverhältnissen sollte ein Hautverschluss nicht erzwungen werden, sondern mit temporären Vakuumversiegelungen gearbeitet werden.

Spätinfekte nach knöcherner Konsolidierung lassen sich in der Regel mit der Durchführung der oben genannten Maßnahmen und einer kompletten Implantatentfernung zur Ausheilung bringen.

Infekte mit Sequesterbildungen erfordern ein radikales Vorgehen mit Entfer- 
nung sämtlichen betroffenen Knochengewebes, eine externe Fixation bei Instabilität und einen mehrzeitigen Gelenkund Knochenaufbau oder die Arthrodese nach Infektausheilung (• Abb. 4g, h; [17]).

\section{Pseudarthrose}

Mit zunehmend operativer Behandlung der Sprunggelenkfrakturen ist die Rate an Falschgelenkbildungen drastisch zurückgegangen. Bei konservativer Gipsimmobilisation betrug die Rate bis $\mathrm{zu}$ $10 \%$, bei Untersuchungen der operierten Patienten liegt die Pseudarthroserate unter $0,2 \%[1,13]$. Gründe für Pseudarthrosen nach operativer Behandlung sind hauptsächlich Infektionen und fehlerhafte bzw. unterlassene Osteosynthesen.

Therapiebedürftig sind die schmerzhaften Pseudarthrosen und die in Fehlstellung stehenden Falschgelenke. Eine straffe schmerzfreie Innenknöchelpseudarthrose bedarf ebenso wenig der operativen Therapie wie eine symptomlose Pseudarthrose nach Weber-A-Fraktur.

Therapeutisch kommen am Innenknöchel die Resektion kleiner Fragmente und die Zuggurtungsosteosynthese bzw. Zugschraubenosteosynthese größerer Fragmente nach Anfrischung des Pseudarthrosespalts zum Einsatz, bei Defektbildung und atrophen Verhältnissen in Kombination mit einem kortikospongiösen Span.

Außenknöchelpseudarthrosen werden nach korrekter Längen- und Rotationseinstellung mit autologen Knochentransplantaten aufgefüllt und osteosynthetisch mit 3,5-mm-LC-DCP oder Rekonstruktionsplatte versorgt. Kleine Spitzenfragmente können analog zum Innenknöchel mittels Zuggurtung zur Ausheilung gebracht werden [23].

Infektpseudarthrosen erfordern eine konsequente Behandlung nach den Prinzipien der Osteitistherapie mit Débridement, Sequestrektomie und lokaler Antibiotikaapplikation, ggf. auch die komplette Segmentresektion sowie ein externes Stabilisationsverfahren. Nach Infektberuhigung sind zum Aufbau der oft entstandenen Defekte Spongiosatransplantationen oder auch Segmenttransporte notwendig,
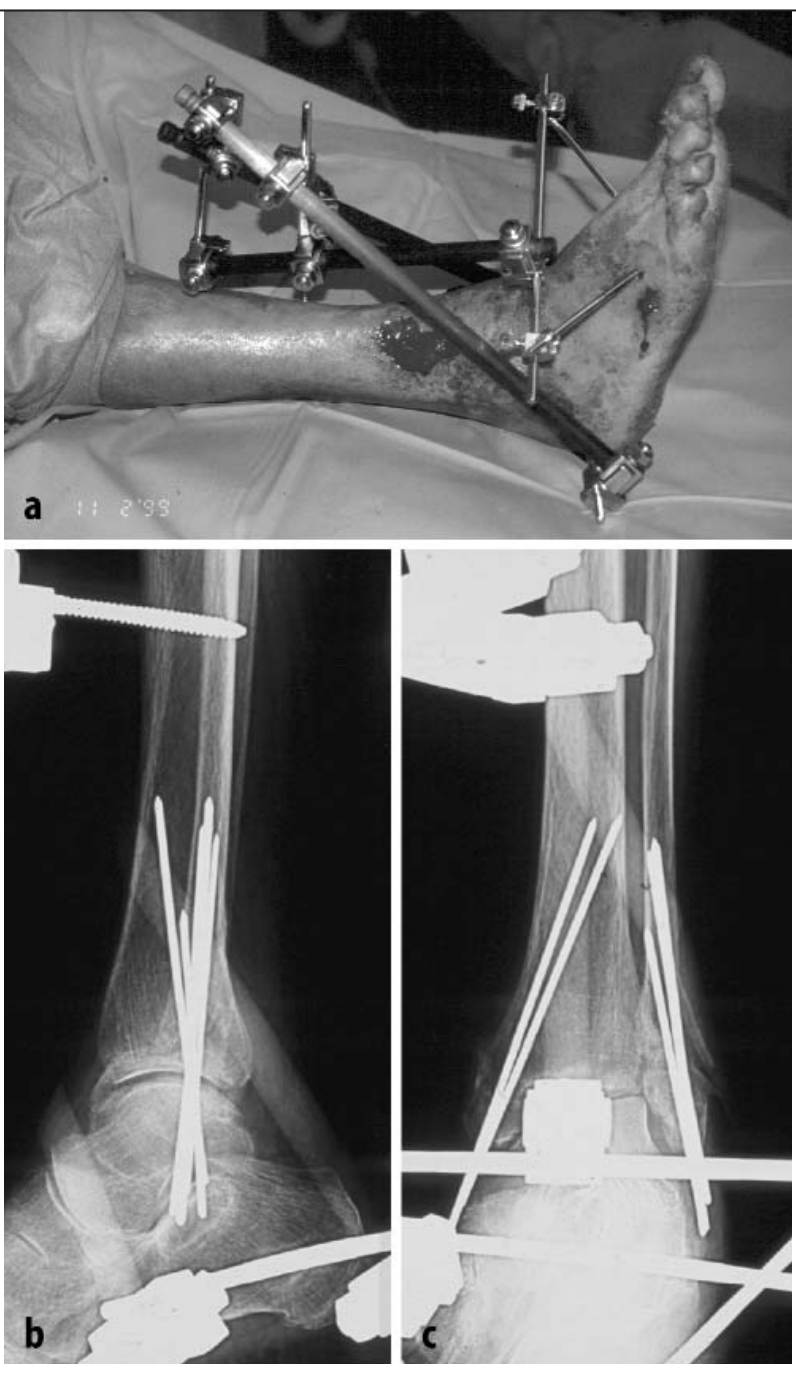

gelegentlich in Kombination mit lokalen Lappenplastiken.

\section{In Fehlstellung verheilte OSG-Frakturen ohne Arthrosezeichen}

Besteht nach verheilter OSG-Fraktur eine korrekturbedürftige Fehlstellung oder eine Instabilität der Knöchelgabel, so sollte diese zügig beseitigt werden, um der posttraumatischen Arthrose vorzubeugen.

Eine präzise und aussagekräftige präoperative bildgebende Diagnostik ist nötig, um das Ausmaß der Fehlstellung genau einzuschätzen. Grundsätzlich sollten Belastungsaufnahmen der Sprunggelenke im Stehen durchgeführt werden. Diese erlauben die Diagnose einer Fibulaverkürzung, liefern wichtige Hinweise für das Vorliegen einer Fehlrotation und einer Syndesmoseninsuffizienz. Auch unter Stufenbildung fehlverheilte Kantendreiecke stellen sich besser dar. Gehaltene Aufnahmen des Sprunggelenkes unter Durchleuchtung liefern wichtige und entscheidende Hinweise auf eine Syndesmoseninsuffizienz. Zusätzlich sollte grundsätzlich eine koronare Computertomographie durchgeführt werden mit zwei- und ggf. dreidimensionalen Rekonstruktionen, um eine präzise Operationsplanung durchführen zu können. Nachfolgende supramalleoläre Fehlstellungen gelten als Indikation für einen Korrektureingriff:

Varus $>5^{\circ}$, Valgus $>10^{\circ}$, Torsion $>10-15^{\circ}$, Ante-Rekurvation $>15^{\circ}$ sowie komplexe Fehlstellungen [21]. Liegen die Fehlstellungen auf Ebene der Gelenkflächen, gelten bereits Fehlstellungen $>5^{\circ}$ in jeder Ebene als korrekturbedürftig, ebenso Verkürzungen der Fibula $>5$ mm und Fehlstellungen der Fibula in der Inzisur von mehr als 2,5 mm im Vergleich zur gesunden Seite [24]. 
Ziel der Rekonstruktion ist ein stabiles, achsengerecht eingestelltes Gelenk mit anatomisch kongruenten Gelenkflächen. Bei der Korrekturosteotomie der distalen Fibula, die abhängig von der gewünschten Korrekturrichtung schräg, quer oder treppenförmig erfolgt, muss die gesamte distale Fibula und die Incisura fibularis tibiae von Pannusgewebe, Fragmenten und Osteophyten befreit werden. Nach korrekter Einstellung werden die Fragmente mit Kleinfragmentrekonstruktionsplatten oder LC-DC-Platten fixiert und Substanzdefekte mit kortikospongiösen Spänen oder autologer Spongiosa aufgefüllt [20].

Die chronische Instabilität des Syndesmosenkomplexes mit pathologischer Weite der Knöchelgabel und rezidivierenden Subluxationsstellungen des Talus stellt ebenfalls eine Indikation zur operativen Korrektur dar. Ziel ist es, eine korrekte dynamische Einstellung der Fibula in ihrer Inzisur zu erreichen. Im Gegensatz zur frischen Verletzung ist hier eine korrekte Einstellung der Fibula und eine temporäre Fixation mit einer Stellschraube nicht ausreichend, da die geschrumpften und vernarbten Bandreste keine dyna- misch-stabile Ausheilung gewährleisten. Erforderlich ist eine rekonstruierende Bandplastik. Die Bandplastik von Castaing mit der Peronaeus-brevis-Sehne wurde von Zwipp et al. im Sinne einer Dreipunktfixierung mit einem distal gestielten Teil der Peronaeus-longus Sehne modifiziert, welche den Verlauf der 3 wichtigsten ligamentären Stabilisatoren des unteren Syndesmosenkomplexes imitiert [2].

\section{Verheilte OSG-Frakturen mit Arthrosezeichen}

Besteht eine ausgeprägte posttraumatische, schmerzhafte Arthrose des oberen Sprunggelenkes, so ist die Arthrodese nach wie vor der ,golden standard“ in der operativen Behandlung. Ziel aller angewanden Verfahren ist, die entknorpelten und sparsam resezierten Gelenkflächen unter Erhalt der Gelenkkontur und in Neutralstellung des Fußes unter Kompression zu bringen und eine schnelle knöcherne Durchbauung zu erreichen. Mehrere Verfahren stehen zur Auswahl. Die Versorgung mit einem bilateralen Kompressionsfixateur ist in den letzten

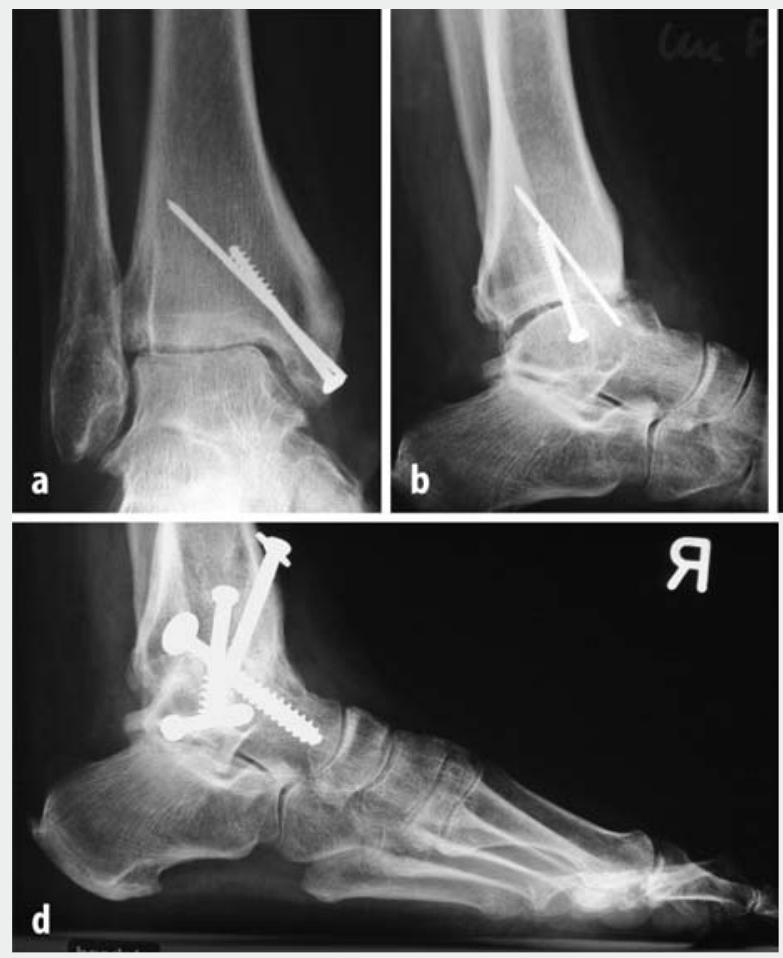

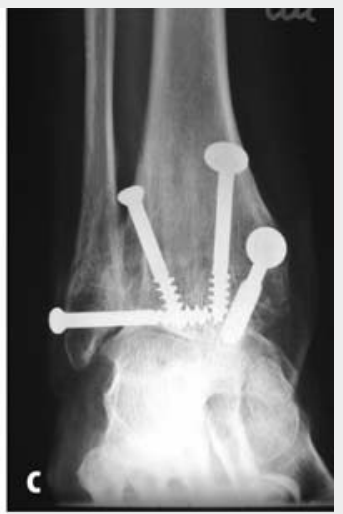

Abb. $7<$ a, b Posttraumatische Arthrose nach operativ behandelter bimalleolärer OSG-Fraktur. $c, d$ Ausheilungsbild der OSG-Arthrodese, die mit 4 kanülierten 7,3 mm-A0Schrauben durchgeführt wurde
Jahren zunehmend verlassen worden zugunsten innerer Stabilisationsverfahren. Nach wie vor haben der Kompressionsfixateur bzw. der Ilizarow-Ringfixateur ihren festen Platz im Behandlungskonzept bei begleitendem Infektgeschehen. Mehrere interne Stabilisierungsverfahren sind etabliert und führen bei korrekter Technik $\mathrm{zu}$ einer hohen Durchbauungsrate, die über 90\% liegt [14].

Das Standardverfahren im eigenen Krankengut ist die von Zwipp beschriebene 4-Schrauben-Technik unter Verwendung von kanülierten 7,3-mmSchrauben (- Abb. 7a-d; [22]). Bei erhaltener Gelenkform kann bei diesem Vorgehen die Entknorpelung der Gelenkflächen des OSG arthroskopisch oder über eine Miniarthrotomie erfolgen. Bei relevanten OSG-Deformitäten und Fehlstellungen des Fußes sollte jedoch ein offenes Vorgehen gewählt werden, um eine korrekte Arthrodeseneinstellung zu gewährleisten und zusätzlich notwendige Span- oder Spongiosaanlagerungen durchführen zu können.

Die Nachteile der OSG-Arthrodesen sind der Bewegungsverlust im OSG, eine Verkürzung des Beines und eine erhebliche Störung des Abrollvorgangs mit Beeinträchtigung des Gangbildes und der Notwendigkeit einer orthopädischen Schuhzurichtung bis hin zum individuellen orthopädischen Maßschuhwerk. Durch die nach durchgeführter OSG-Arthrodese teilweise übermäßigen unphysiologischen Belastungen der Nachbargelenke kommt es trotz korrekt durchgeführter Arthrodese zu Anschlussarthrosen. Lattig et al. sahen bei 30 nachuntersuchten OSG-Arthrodesen nach durchschnittlich 23,5 Jahren zwar 55\% gute und sehr gute klinische Ergebnisse, jedoch wiesen fast $90 \%$ der Rück- und Mittelfüße begleitende arthrotische Veränderungen auf [9]. Diese Nachbararthrosen können dann weitere Arthrodesen notwendig machen, die eine zunehmende Rigidität des Fußes verursachen und zu erheblichen Gehbehinderungen führen.

Diese Problematik und der Wunsch, ein funktionsfähiges oberes Sprunggelenk zu erhalten, führt die Diskussion immer wieder in Richtung des endoprothetischen Gelenkersatzes. Die Berichte über die erstentwickelten uniaxialen reinen Schar- 

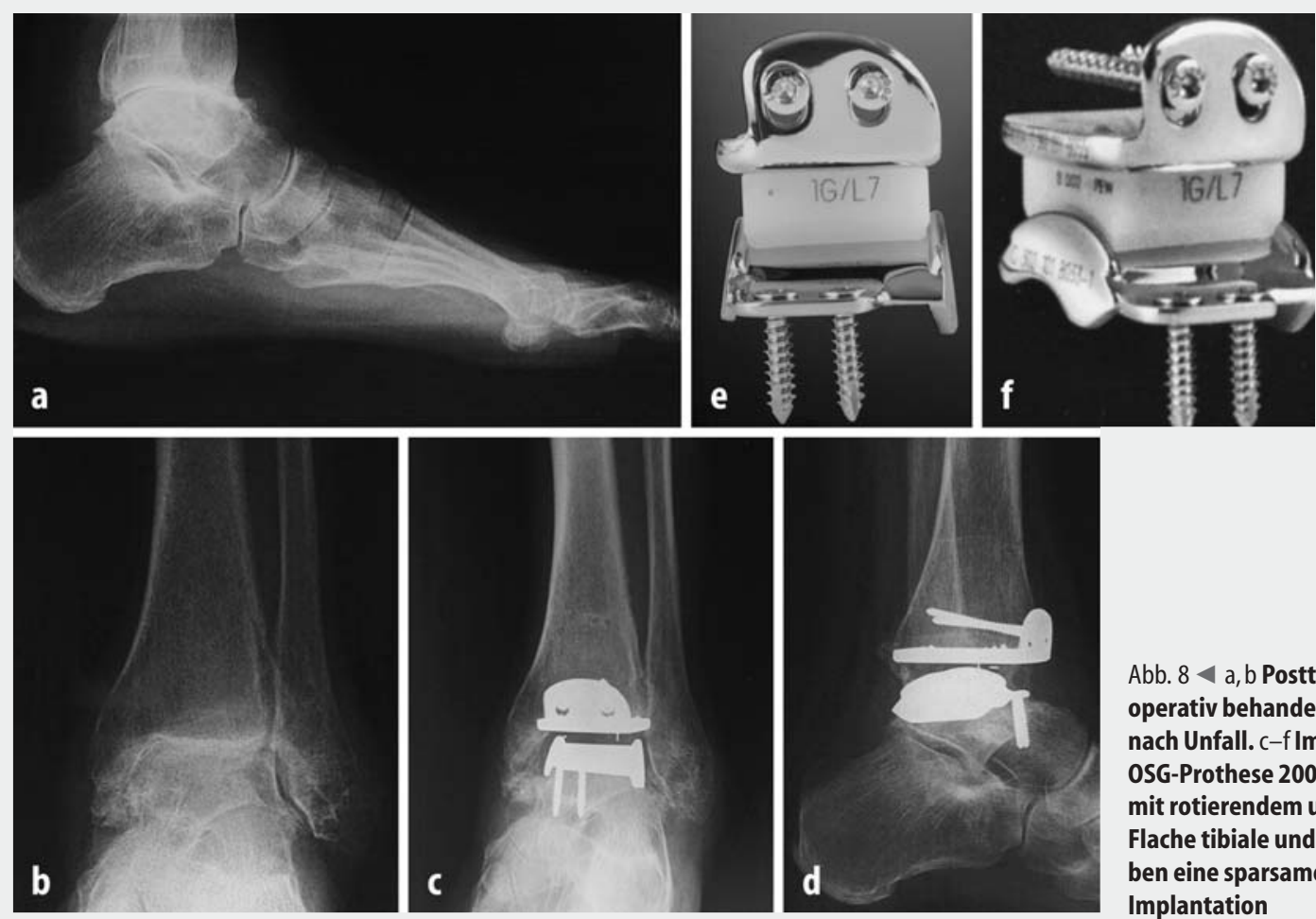

Abb. $8<$ a, b Posttraumatische Arthrose nach operativ behandelter OSG-Fraktur 13 Jahre nach Unfall. c-f Implantation einer "Hintegra"OSG-Prothese 2003. Dreikomponentendesign mit rotierendem und gleitendem PE-Inlay. Flache tibiale und talare Auflageflächen erlauben eine sparsame Knochenresektion bei der Implantation

nierprothesen und biaxialen Gelenke sind ernüchternd und die Langzeitergebnisse denen der OSG-Arthrodesen deutlich unterlegen. Über hohe Lockerungsraten und Instabilitätsprobleme wurde berichtet [8]. Mit der Weiterentwicklung der Prothesen hin zum Dreikomponentenprinzip mit einer die Meniskusfunktion imitierenden gleitenden und rotierenden PEZwischenscheibe nähern sich die Prothesen in ihrem Bewegungsablauf der komplexen dreidimensionalen OSG-Mechanik. Diese Prothesentypen zeigen in ersten klinischen Studien größtenteils bessere kurz- und mittelfristige Ergebnisse.

Uneinheitlich stellen sich die bisher verfügbaren Ergebnisse der neuen Dreikomponenten-OSG-Prothesen bei der posttraumatischen Arthrose dar. Sahen Sailer et al. in einer kleinen Serie 50\% Lockerungsraten und rieten von der Implantation des gewählten Prothesentyps ab, so berichten Hagena et al. bei einer gemischten Klientel von 76 Patienten mit einem Anteil von 40,9\% posttraumatischen Arthrosen bei gleichem Prothesentyp über deutlich bessere Zahlen nach durchschnittlich 3,6 Jahren Standzeit [4, 15]. Ähnlich wie auch in der Hüft- und Kniegelenkprothetik sind die Ergebnisse nach
OSG-Prothesen in Bezug auf Bewegungsgewinn und Schmerzreduktion bei Patienten mit posttraumatischer Arthose schlechter als bei den Patienten mit idiopathischer Arthrose [6].

Wir sehen die Indikation bei posttraumatischer Arthrose zur Prothesenimplantation, wenn der Patient noch über eine ausreichende Bewegungsfreiheit im oberen Sprunggelenk verfügt, die Knochensubstanz gut ist und das Alignement des Fußes weitgehend erhalten ist (- Abb. 8a-d). Weiterhin muss der Patient ausführlich und mehrzeitig aufgeklärt werden und bereit sein, das Risiko der Lockerung und eines versteifenden Zweiteingriffs zu tragen. Kontraindikationen sind ausgeprägte Instabilitäten, schwere OSG-Fehlstellungen, Osteonekrosen, Begleiterkrankungen in Form einer pAVK oder Mikroangiopathie sowie eine mangelnde Patientencompliance.

Lockert eine Sprunggelenkprothese aus, so bleibt als weiterer Schritt, abgesehen von der Option des Wechseleingriffs, die Explantation der Arthroplastik und Einstellung einer Arthrodese in der zuvor ausgeführten Technik (• Abb. 9a-d). Im gezeigten Beispiel führt dieses Vorgehen zu einer deutliche Verkürzung des be- troffenen Beines. Wir verwenden daher ein Prothesenmodell, welches nur eine sparsame Knochenresektion erfordert und zementfrei zu verankern ist. Hierdurch ist gewährleistet, dass bei Lockerung der Prothese ohne relevante Beinverkürzung nach Explantation eine OSGArthrodese durchgeführt werden kann.

Die Endoprothetik der posttraumatischen OSG-Arthrodese kann aus den genannten Gründen noch nicht als Standardverfahren empfohlen werden und sollte Zentren vorbehalten sein, die über die Erfahrungswerte verfügen sowie wissenschaftliche Nachuntersuchungen und Ergebnisaufarbeitungen der Daten gewährleisten können.

\section{Fazit}

Die Prognose nach OSG-Frakturen wird durch unterschiedliche Faktoren bestimmt. Nicht zu beeinflussen sind Schwere und Ausmaß der knöchern-ligamentären Verletzung und der begleitenden Knorpelläsionen. Ein konsequentes perioperatives Weichteilmanagement schafft die Voraussetzungen für eine ungestörte Heilung und reduziert die Infektraten. Eine standardisierte und selbstkritische postoperative Ergebniskontrolle hilft, 


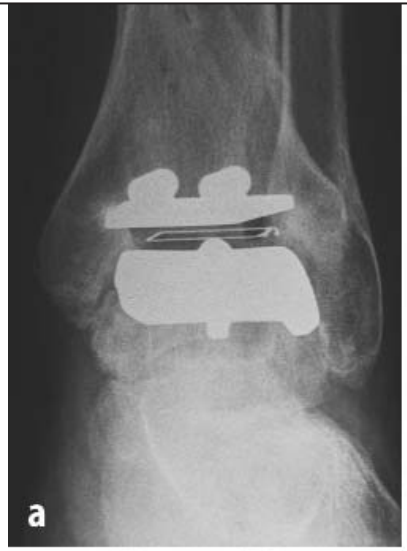

\section{\begin{tabular}{l|l} 
Sprunggelenkfrakturen \\
\hline
\end{tabular}}
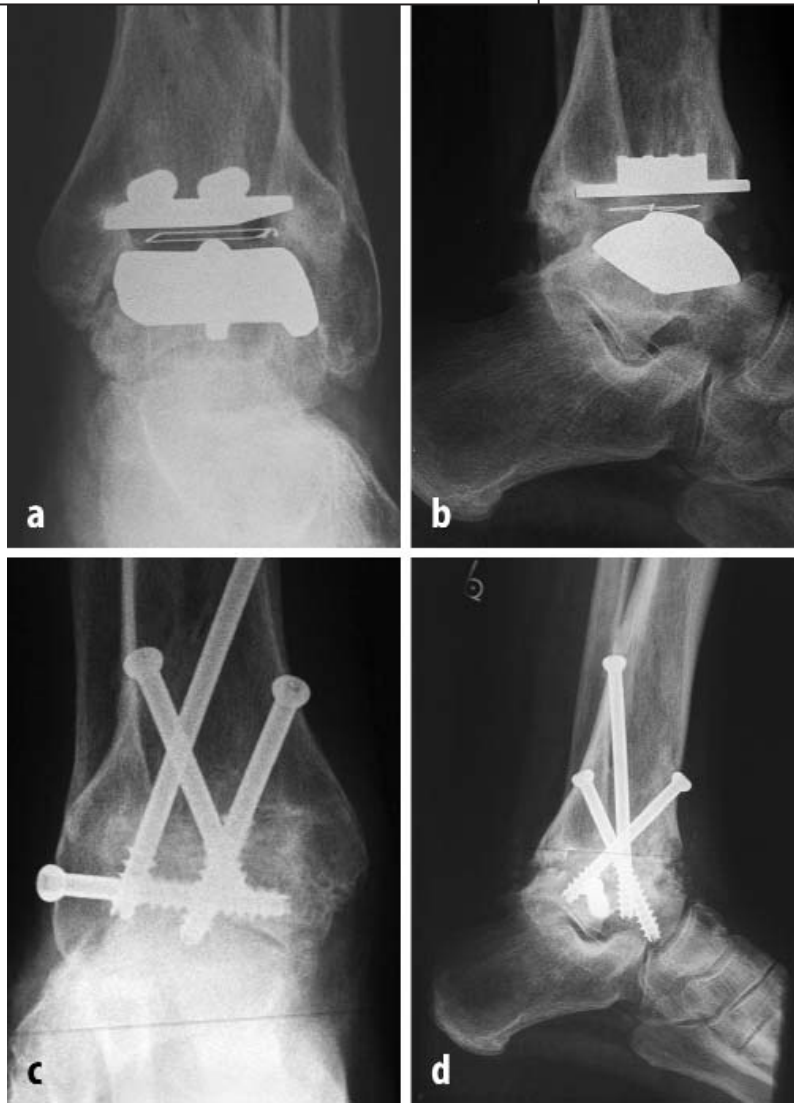

Abb. $9<$ a, b Gelockerte Sprunggelenkprothese 4 Jahre nach Implantation wegen posttraumatischer Arthrose. $c, d$ Prothesenausbau, 0SG-Arthrodese mit 4 kanülierten 7,3-mmA0-Schrauben in der Technik nach Zwipp fehlerhafte Versorgungen rechtzeitig zu erkennen und zu korrigieren. Gerade die oft vernachlässigten begleitenden tibiofibularen Bandverletzungen des unteren Syndesmosenkomplexes bei Maisonneuve-Verletzungen und Pronations-Eversions-Frakturen erfordern aus unserer Sicht eine routinemäßige postoperative Computertomographie, da die konventionelle Bildgebung Fehlstellungen der Fibula nicht ausreichend objektivieren kann.

"Golden standard“ für die Behandlung der posttraumatischen Arthrose ist die Arthrodese des oberen Sprunggelenks. Wir bevorzugen ein patientenkomfortables internes Stabilisationsverfahren und verwenden den Fixateur in der llizarov-Ringtechnik nur noch bei begleitender Infektsymptomatik. Die OSG-Prothetik bei posttraumatischer Arthrose zeigt zunehmend bessere Ergebnisse, erfordert aber eine genaue Indikationsstellung und sollte zum jetzigen Zeitpunkt Zentren vorbehalten sein, die über ausreichende Erfahrungswerte verfügen und regelmäßige Nachuntersuchungen und Datenaufarbeitung gewährleisten können.

\section{Korrespondierender Autor \\ Dr. M. Beck}

Abteilung für Unfall- und Wiederherstellungschirurgie der Klinik und Poliklinik für Chirurgie, Universität Rostock,

Schillingallee 35, 18057 Rostock

E-mail: markus.beck@med.uni-rostock.de

Interessenkonflikt: Der korrespondierende Autor versichert, dass keine Verbindungen mit einer Firma, deren Produkt in dem Artikel genannt ist, oder einer Firma, die ein Konkurrenzprodukt vertreibt, bestehen.

\section{Literatur}

1. Bauer M, Jonsson K, Nilsson B (1985) Thirty-year followup of ankle fractures. Acta Orthop Scand 56: 103-106

2. Grass R, Zwipp H (2003) Syndesmosenplastik bei chronischer Insuffizienz des distalen tibiofibularen Syndesmosenkomplexes. Operat Orthop Traumatol 15: 208225

3. Grass R, Herzmann K, Biewener A, Zwipp H (2000) Verletzungen der unteren tibiofibularen Syndesmose. Unfallchirurg 103: 520-532

4. Hagena F-W, Christ R, Kettrukat M (2003) Die Endoprothese am oberen Sprunggelenk. Fuss Sprungg 1: 48-55

5. Hintermann B, Regazzoni P, Lampert C, Stutz G, Gächter A (2000) Arthroscopic findings in acute fractures of the ankle. J Bone Joint Surg Br 82: 345-351

6. Hintermann B (1999) Die Star-Sprunggelenkprothese. Orthopäde 28: 792-803
7. Joseph J,Giannoudis PV, Hinsche A et al. (2000) Compartment syndrome following isolated ankle fracture. Int Orthop 24: 173-175

8. Kitaoka HB, Patzer GL (1996) Clinical results of the MAYO total ankle arthroplasty.J Bone Joint Surg Am 78: 1658-1664

9. Lattig CF, Kuster MS, Lampert C (2003) Does foot position in tibiotalar arthrodesis have an effect on development of secondary arthrosis. Foot Ankle Surg 9: 25-29

10. Lorez LG, Hintermenn B (1999) Bedeutung von Begleitverletzungen bei Malleolarfrakturen. Unfallchirurg 102: 949-954

11. Meyer-Wölbert B, Schmidt R, Benesch S, Fels T, Becker HP (1999) Die prognostische Bedeutung verletzter Anteile bei Sprunggelenkfrakturen. Chirurg 70: 13231329

12. Müller ME, Allgöwer $M$, Schneider R, Willenegger $H$ (1992) Manual der Osteosynthese. Springer, Berlin Heidelberg New York Tokio

13. Nigst H (1961) Über Malleolarfrakturen: PronationsDorsalflexions-Frakturen, Bedeutung des hinteren Volkmannschen Dreiecks, Pseudarthrosen des Malleolus tibialis. Monatsschr Unfallheilkd 64: 351-357

14. Rowan R, Davey KJ (1999) Ankle arthrodesis using an anterior A0 T plate.J Bone Joint Surg Br 81: 113-116

15. Sailer R, HackI W, Klestil T et al. (2002) Totalendoprothese des oberen Sprunggelenks nach posttraumatischer Arthrose. Unfallchirurg 105: 170-173

16. Stürmer KM (Hrsg) (1999) Leitlinien Unfallchirurgie. Sprunggelenksfraktur, 2. Aufl. Thieme, Stuttgart, S 197207

17. Szyszkowitz R (Hrsg) (2003) Unterschenkel. In: Tscherne $\mathrm{H}$ (Hrsg) Unfallchirurgie, Bd 9. Springer, Berlin Heidelberg New York Tokio, S 115-147

18. Thordarson DB, Motamed S, Hedman T, Ebramzadeh E, Bakshian S (1997) The effect of fibular malreduction on contact pressures in an ankle fracture malunion model. J Bone Joint Surg Am 79: 1809-1815

19. Vandergriend R, Michelson JD, Larry B (1996) Ankle fractures. An instructional course lecture. The American Academy of Orthopaedic Surgeons.J Bone Joint Surg Am 78: 1772-1783

20. Weber D, Fritschy D, Friederich NF, Müller W (2001) Korrekturosteotomie der distalen Fibula bei posttraumatischer Fehlstellung. Operat Orthop Traumatol 13: 292304

21. Weise K, Weller S (1997) Supramalleoläre Korrekturosteotomien. In: Strecker W, Keppler P, Kinzl L (Hrsg) Posttraumatische Beindeformitäten: Analyse und Korrektur.Springer, Berlin Heidelberg New York Tokio, S 215-220

22. Zwipp H (1994) Chirurgie des Fußes. Springer, Berlin Heidelberg New York Tokio, S 188-192

23. Zwipp H, Franck WM, Tscherne H (1996) Pseudarthrosen im Bereich des oberen Sprunggelenks. Orthopäde 25: 463-469

24. Zwipp H, Grass R (2003) Rekonstruktion nach Fehlheilung der Pilon-tibiale- und oberen Sprunggelenkfraktur. Trauma Berufskrankh 5 [Suppl 2]: S285-S289 